\title{
Bottomonium Studies at Belle
}

\author{
A. Garmash ${ }^{1,2, a}$ \\ ${ }^{1}$ Budker Institute of Nuclear Physics \\ ${ }^{2}$ Novosibirsk State University
}

\begin{abstract}
Recent results on studies of bottomonium and bottomonium-like states at Belle are reported. The results are obtained with a $121.4 \mathrm{fb}^{-1}$ data sample collected with the Belle detector in the vicinity of the $\Upsilon(5 S)$ resonance at the KEKB asymmetric-energy $e^{+} e^{-}$collider.
\end{abstract}

\section{Introduction}

Bottomonium is the bound system of $b \bar{b}$ quarks and is considered to be an excellent laboratory to study Quantum Chromodynamics (QCD) at low energies. Due to relatively large mass of the $b$ quark, the system is essentially non-relativistic, thus the quark-antiquark QCD potential can be investigated via the $b \bar{b}$ spectroscopy.

Analysis of spin-singlet states $h_{b}(m P)$ and $\eta_{b}(n S)$ provides information on the spin-spin interaction in the bottomonium system. Hyperfine splitting is the difference between the spin-weighted average mass of the $P$-wave triplet states $\chi_{b J}(n P)\left(n^{3} P_{J}\right.$ state $)$ and that of the corresponding $h_{b}(m P)$, or $m^{1} P_{1}$. These splittings are predicted to be close to zero [1], and recent precision measurements of the $h_{c}(1 P)$ mass validate this expectation for charmonium.

The CLEO Collaboration has recently measured the cross section of the $e^{+} e^{-} \rightarrow h_{c}(1 P) \pi^{+} \pi^{-}$process to be comparable to that of the $e^{+} e^{-} \rightarrow J / \psi \pi^{+} \pi^{-}$in data taken above open charm threshold [2]. Such a strong enhancement in the rate contradicts to expectations as the production of the $h_{c}(1 P)$ requires a $c$-quark spin flip, while production of the $J / \psi$ does not. Similarly, the Belle Collaboration reported anomalously high rates for $e^{+} e^{-} \rightarrow$ $\Upsilon(n S) \pi^{+} \pi^{-}(n=1,2,3)$ process at energies near the $\Upsilon(5 S)$ mass [3]. Combined, these observations motivated a more detailed study of bottomonium production at the c.m. energy near the peak of $\Upsilon(5 S)$ resonance.

We use a $121.4 \mathrm{fb}^{-1}$ data sample collected on or near the peak of the $\Upsilon(5 S)$ resonance $(\sqrt{s} \sim 10.865 \mathrm{GeV})$ with the Belle detector [4] at the KEKB asymmetric-energy $e^{+} e^{-}$collider [5].

\section{Observation of $h_{b}(m P)$}

As mentioned above, the CLEO Collaboration observed strong enchancement in rates of both $e^{+} e^{-} \rightarrow h_{c}(1 P) \pi^{+} \pi^{-}$

\footnotetext{
ae-mail: garmash@inp.nsk.su
}

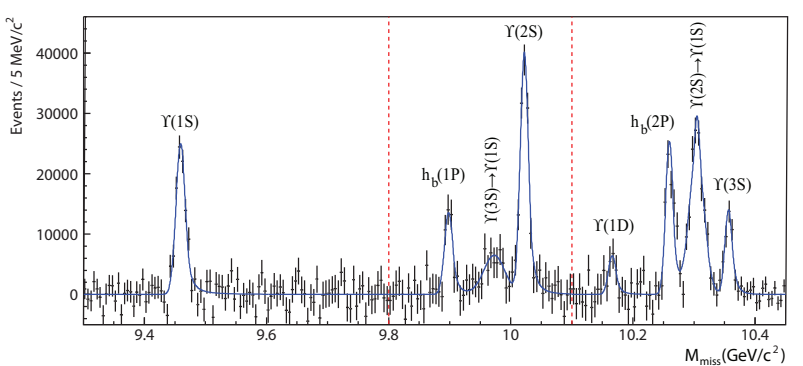

Figure 1. The inclusive $M_{\text {miss }}\left(\pi^{+} \pi^{-}\right)$spectrum with the combinatorial background and $K_{S}^{0}$ contribution subtracted (points with errors) and signal component of the fit function overlaid (smooth curve). The vertical lines indicate boundaries of the fit regions.

and $e^{+} e^{-} \rightarrow J / 4 \pi^{+} \pi^{-}$processes. Based on the observation of the anomalously large rate of the $e^{+} e^{-} \rightarrow \Upsilon(n S) \pi^{+} \pi^{-}$ process in the vicinity of the $\Upsilon(5 S)$ resonance by the Belle Collaboration, we decided to extend the analysis to measure the rate of the $e^{+} e^{-} \rightarrow h_{b}(m P) \pi^{+} \pi^{-}$process. As there is no dominant $h_{b}(m P)$ decay channel, we use inclusive reconstruction.

A set of requirements applied to select hadronic events includes a well reconstructed primary vertex, consistent with the run-averaged interaction point and at least three high-quality charged tracks. The $\pi^{+} \pi^{-}$candidates are all pairs of oppositely charged tracks that are identified as pions and are not consistent with being electrons. Continuum $e^{+} e^{-} \rightarrow q \bar{q}(q=u, d, s, c)$ background is suppressed utilizing event topology variables: the ratio of the second to zeroth Fox-Wolfram moments is required to be $R_{2}<0.3$. More details can be found in Ref. [6].

We calculate missing mass defined as $M_{\text {miss }}\left(\pi^{+} \pi^{-}\right) \equiv$ $\sqrt{\left(P_{\Upsilon(5 S)}-P_{\pi^{+} \pi^{-}}\right)^{2}}$, where $P_{e^{+} e^{-}}$and $P_{\pi^{+} \pi^{-}}$are 4-momenta of the initial $e^{+} e^{-}$system and $\pi^{+} \pi^{-}$system, respectively. The $M_{\text {miss }}\left(\pi^{+} \pi^{-}\right)$spectrum is divided into three adjacent regions with boundaries at $M_{\text {miss }}\left(\pi^{+} \pi^{-}\right)=9.3,9.8$, 10.1 and $10.45 \mathrm{GeV} / c^{2}$ and fitted separately in each re- 
Table 1. The yield and mass determined from the fits to the $M_{\text {miss }}\left(\pi^{+} \pi^{-}\right)$distributions.

\begin{tabular}{lcc}
\hline \hline & Yield, $10^{3}$ & Mass, MeV $/ c^{2}$ \\
\hline$\Upsilon(1 S)$ & $105.2 \pm 5.8 \pm 3.0$ & $9459.4 \pm 0.5 \pm 1.0$ \\
$h_{b}(1 P)$ & $50.4 \pm 7.8_{-9.1}^{+4.5}$ & $9898.3 \pm 1.1_{-1.1}^{+1.0}$ \\
$3 S \rightarrow 1 S$ & $56 \pm 19$ & 9973.01 \\
$\Upsilon(2 S)$ & $143.5 \pm 8.7 \pm 6.8$ & $10022.3 \pm 0.4 \pm 1.0$ \\
$\Upsilon(1 D)$ & $22.0 \pm 7.8$ & $10166.2 \pm 2.6$ \\
$h_{b}(2 P)$ & $84.4 \pm 6.8_{-10 .}^{+23 .}$ & $10259.8 \pm 0.6_{-1.0}^{+1.4}$ \\
$2 S \rightarrow 1 S$ & $151.7 \pm 9.7_{-20 .}^{+9.0}$ & $10304.6 \pm 0.6 \pm 1.0$ \\
$\Upsilon(3 S)$ & $45.6 \pm 5.2 \pm 5.1$ & $10356.7 \pm 0.9 \pm 1.1$ \\
\hline \hline
\end{tabular}

gion. In the third region, prior to fitting, we perform bin-by-bin subtraction of the background associated with the $K_{S}^{0} \rightarrow \pi^{+} \pi^{-}$production. The combinatorial background is parametrized by a Chebyshev polynomial function (6th order for the first two regions and 7th order for the third region). The signal component consists of three $\Upsilon(n S)$ signals, two $h_{b}(m P)$ signals, $\Upsilon(1 D)$ as well as feed across from the $\Upsilon(2 S) \rightarrow \Upsilon(1 S) \pi^{+} \pi^{-}$and $\Upsilon(3 S) \rightarrow$ $\Upsilon(1 S) \pi^{+} \pi^{-}$transitions. The peak positions of all signals are floated, except that for $\Upsilon(3 S) \rightarrow \Upsilon(1 S) \pi^{+} \pi^{-}$, which is poorly constrained by the fit. The background subtracted $M_{\text {miss }}\left(\pi^{+} \pi^{-}\right)$distribution is shown in Fig. 1, where signals of both $h_{b}(1 P)$ and $h_{b}(2 P)$ are clearly visible. The signal parameters are listed in Table 1. Statistical significance of all signals except that for the $\Upsilon(1 D)$ exceeds $5 \sigma$.

The measured masses of $h_{b}(1 P)$ and $h_{b}(2 P)$ are $M=\left(9898.3 \pm 1.1_{-1.1}^{+1.0}\right) \mathrm{MeV} / c^{2}$ and $M=(10259.8 \pm$ $\left.0.6_{-1.0}^{+1.4}\right) \mathrm{MeV} / c^{2}$, respectively. Using the world average masses of the $\chi_{b J}(n P)$ states, we determine the hyperfine splittings to be $\Delta M_{\mathrm{HF}}=(+1.6 \pm 1.5) \mathrm{MeV} / c^{2}$ and $\left(+0.5_{-1.2}^{+1.6}\right) \mathrm{MeV} / c^{2}$, respectively, where statistical and systematic uncertainties are combined in quadrature.

We also measure the ratio of cross sections $R \equiv$ $\frac{\sigma\left(h_{b}(m P) \pi^{+} \pi^{-}\right)}{\sigma\left(\Upsilon(2 S) \pi^{+} \pi^{-}\right)}$. To determine the reconstruction efficiency we use the results of resonant structure studies reported below. We find the ratio of cross sections to be $R=$ $0.46 \pm 0.08_{-0.12}^{+0.07}$ for the $h_{b}(1 P)$ and $R=0.77 \pm 0.08_{-0.17}^{+0.22}$ for the $h_{b}(2 P)$.

\section{Observation of $Z_{b}\left(10610\right.$ and $Z_{b}(10650)$}

As shown above, the rates of the processes $\Upsilon(5 S) \rightarrow$ $h_{b}(m P) \pi^{+} \pi^{-}(m=1,2)$, that require a heavy quark spin flip, are found to be comparable to those for the heavy quark spin conserving transitions $\Upsilon(5 S) \rightarrow \Upsilon(n S) \pi^{+} \pi^{-}$, $(n=1,2,3)$. This observation contradicts to a priori theoretical expectations and strongly suggests that some exotic mechanisms are contributing to $\Upsilon(5 S)$ decays.

To study the resonant substructure of the $\Upsilon(5 S) \rightarrow$ $h_{b}(m P) \pi^{+} \pi^{-}(m=1,2)$ three-body decays, we measure the $h_{b}(m P)$ yield as a function of the $h_{b}(m P) \pi^{ \pm}$invariant mass. We use the same inclusive approach as described in the previous section. We fit the $M_{\text {miss }}\left(\pi^{+} \pi^{-}\right)$spectrum in bins of $h_{b}(m P) \pi^{ \pm}$invariant mass, defined as the missing mass to the opposite sign pion, $M_{\text {miss }}\left(\pi^{\mp}\right)$. We combine the
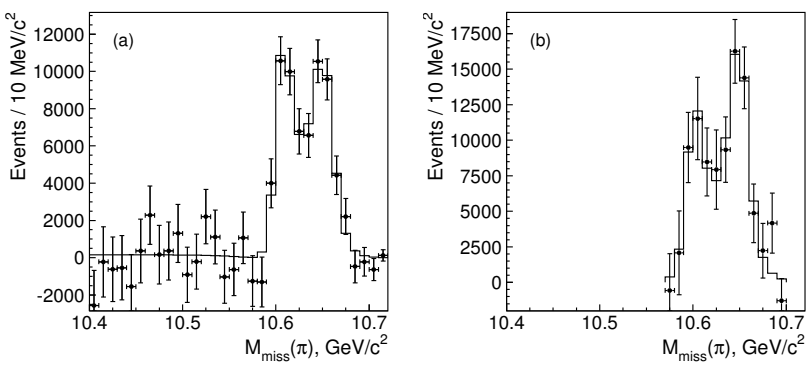

Figure 2. The (a) $h_{b}(1 P)$ and (b) $h_{b}(2 P)$ yields as a function of $M_{\text {miss }}(\pi)$ (points with error bars) and results of the fit (histogram).

$M_{\text {miss }}\left(\pi^{+} \pi^{-}\right)$spectra for the corresponding bin in $M_{\text {miss }}\left(\pi^{-}\right)$ and $M_{\text {miss }}\left(\pi^{+}\right)$and use half of the available $M_{\text {miss }}(\pi)$ range to avoid double counting.

The results for the yield of $\Upsilon(5 S) \rightarrow h_{b}(m P) \pi^{+} \pi^{-}$ ( $m=1,2)$ decays as a function of the $M_{\text {miss }}(\pi)$ are shown in Fig. 2. The distribution for the $h_{b}(1 P)$ exhibits a clear two-peak structure without a significant non-resonant contribution. A similar distribution is observed for the $h_{b}(2 P)$ yield, though the available phase space is much smaller. In the following we refer to these structures as $Z_{b}(10610)$ and $Z_{b}(10650)$, respectively. To fit the $M_{\text {miss }}(\pi)$ spectrum we use the following combination:

$$
\left|B W_{1}\left(s, M_{1}, \Gamma_{1}\right)+a e^{i \phi} B W_{1}\left(s, M_{2}, \Gamma_{2}\right)+b e^{i \psi}\right|^{2} \frac{q p}{\sqrt{s}} .
$$

Here $\sqrt{s} \equiv M_{\text {miss }}(\pi)$; the variables $M_{k}, \Gamma_{k}(k=1,2)$, $a, \phi, b$ and $\psi$ are free parameters; $\frac{q p}{\sqrt{s}}$ is a phase-space factor, where $p(q)$ is the momentum of the pion originating from the $\Upsilon(5 S)\left(Z_{b}\right)$ decay measured in the rest frame of the corresponding mother particle. The $P$-wave Breit-Wigner amplitude is expressed as $B W_{1}(s, M, \Gamma)=$ $\frac{\sqrt{M \Gamma} F\left(q / q_{0}\right)}{M^{2}-s-i M \Gamma}$. Here $F$ is the $P$-wave Blatt-Weisskopf form factor $F=\sqrt{\frac{1+\left(q_{0} R\right)^{2}}{1+(q R)^{2}}}, q_{0}$ is a daughter momentum calculated with pole mass of its mother, $R=1.6 \mathrm{GeV}^{-1}$. The function (Eq. 1) is convolved with the detector resolution function, integrated over the histogram bin and corrected for the reconstruction efficiency. The fit results are shown as solid histograms in Fig. 2. We find that the non-resonant contribution is consistent with zero in accord with the expectation that it is suppressed due to heavy quark spin-flip. In case of the $h_{b}(2 P)$ we fix the non-resonant amplitude at zero.

Another possibility to study the observed $Z_{b}$ states is provided by the $e^{+} e^{-} \rightarrow \Upsilon(n S) \pi^{+} \pi^{-}, n=1,2,3$ process. The observed rates for these processes are about two orders of magnitude higher than expected. To study these processes we use a full reconstruction approach that allows to reduce the background significantly.

To reconstruct $\Upsilon(5 S) \rightarrow \Upsilon(n S) \pi^{+} \pi^{-}, \Upsilon(n S) \rightarrow \mu^{+} \mu^{-}$ candidates, we select events with four charged tracks with zero net charge that are consistent with coming from the interaction point. Charged pion and muon candidates are required to be positively identified. Candidate $\Upsilon(5 S) \rightarrow \Upsilon(n S) \pi^{+} \pi^{-}$events are selected by requiring $\left|M_{\text {miss }}\left(\pi^{+} \pi^{-}\right)-m_{\Upsilon(n S)}\right|<0.05 \mathrm{GeV} / c^{2}$, where $m_{\Upsilon(n S)}$ 

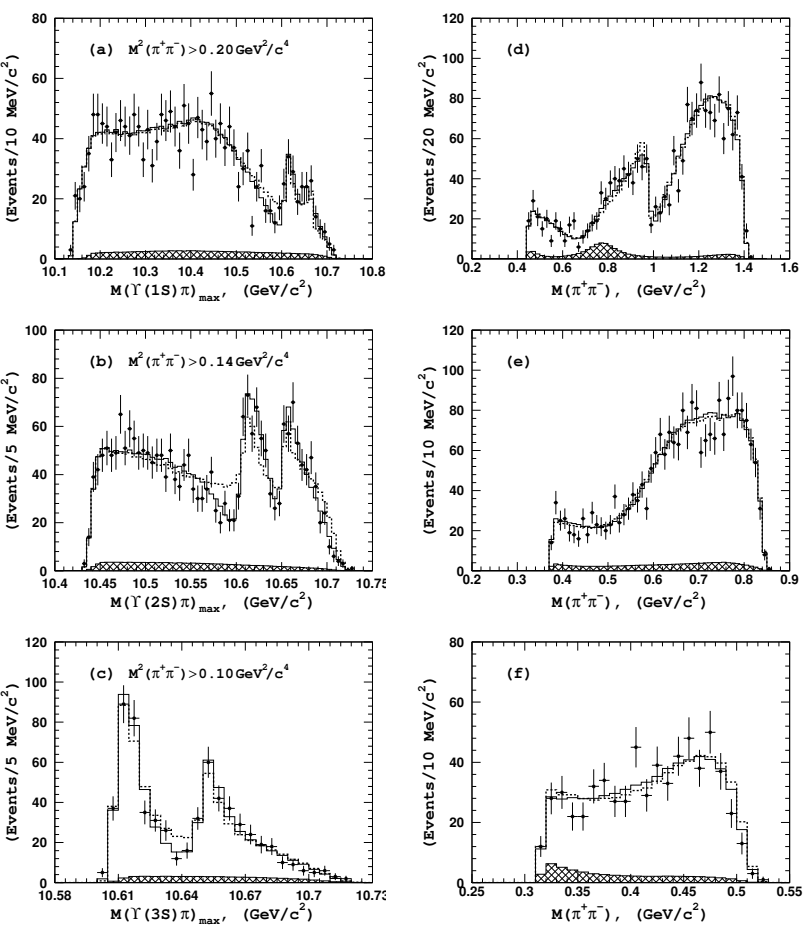

Figure 3. Comparison of fit results (open histogram) with experimental data (points with error bars) for events in the $\Upsilon(1 S)$ (top), $\Upsilon(2 S)$ (middle), and $\Upsilon(3 S)$ (bottom) signal regions. The solid histogram is for $J^{P}=1^{+}$model, dashed one is for $J^{P}=2^{+}$model for $Z_{b}$ states. The hatched histogram shows the background component.

is the mass of an $\Upsilon(n S)$ state [7]. Sideband regions are defined as $0.05 \mathrm{GeV} / c^{2}<\left|M_{\text {miss }}\left(\pi^{+} \pi^{-}\right)-m_{\Upsilon(n S)}\right|<$ $0.10 \mathrm{GeV} / c^{2}$. To remove background due to photon conversions on the innermost parts of the Belle detector, we require $M^{2}\left(\pi^{+} \pi^{-}\right)>0.20 / 0.14 / 0.10 \mathrm{GeV} / c^{2}$ for a final state with an $\Upsilon(1 S), \Upsilon(2 S)$, and $\Upsilon(3 S)$, respectively. More details can be found in Ref. [8].

One-dimensional invariant mass distributions for events in the $\Upsilon(n S)$ signal regions are shown in Fig. 3, where two peaks are evident in the $\Upsilon(n S) \pi$ system near $10.61 \mathrm{GeV} / c^{2}$ and $10.65 \mathrm{GeV} / c^{2}$.

Amplitude analyses are performed by means of unbinned maximum likelihood fits to six-dimensional phase space [9]. The variation of reconstruction efficiency across the Dalitz plot is determined from a GEANT-based MC simulation. The distribution of background events is determined using sideband events and found to be uniform across the Dalitz plot.

We parametrize the $\Upsilon(5 S) \rightarrow \Upsilon(n S) \pi^{+} \pi^{-}$three-body decay amplitude by:

$$
M=A_{Z_{1}}+A_{Z_{2}}+A_{\sigma}+A_{f_{0}}+A_{f_{2}}+A_{\mathrm{nr}},
$$

where $A_{Z_{1}}$ and $A_{Z_{2}}$ are amplitudes to account for contributions from the $Z_{b}(10610)$ and $Z_{b}(10650)$, respectively.

Consequently, we parametrize the observed $Z_{b}(10610)$ and $Z_{b}(10650)$ peaks with an $S$-wave Breit-Wigner function $B W(s, M, \Gamma)=\frac{\sqrt{M \Gamma}}{M^{2}-s-i M \Gamma}$, where we do not consider possible $s$-dependence of the resonance width. To account for the possibility of $\Upsilon(5 S)$ decay to both $Z_{b}^{+} \pi^{-}$and $Z_{b}^{-} \pi^{+}$, the amplitudes $A_{Z_{1}}$ and $A_{Z_{2}}$ are symmetrized with respect to $\pi^{+}$and $\pi^{-}$transposition. Using isospin symmetry, the resulting amplitude is written as

$$
A_{Z_{k}}=a_{Z_{k}} e^{i \delta_{Z_{k}}}\left(B W\left(s_{1}, M_{k}, \Gamma_{k}\right)+B W\left(s_{2}, M_{k}, \Gamma_{k}\right)\right),
$$

where $s_{1}=M^{2}\left[\Upsilon(n S) \pi^{+}\right], s_{2}=M^{2}\left[\Upsilon(n S) \pi^{-}\right]$. The relative amplitudes $a_{Z_{k}}$, phases $\delta_{Z_{k}}$, masses $M_{k}$ and widths $\Gamma_{k}$ $(k=1,2)$ are free parameters. We also include the $A_{f_{0}}$ and $A_{f_{2}}$ amplitudes to account for possible contributions in the $\pi^{+} \pi^{-}$channel from the $f_{0}(980)$ scalar and $f_{2}(1270)$ tensor states, respectively. We use a Breit-Wigner function to parametrize the $f_{2}(1270)$ and a coupled-channel Breit-Wigner function for the $f_{0}(980)$. The mass and width of the $f_{2}(1270)$ state are fixed at their world average values [7]; the mass and the coupling constants of the $f_{0}(980)$ state are fixed at values determined from the analysis of $B^{+} \rightarrow K^{+} \pi^{+} \pi^{-}: M\left[f_{0}(980)\right]=950 \mathrm{MeV} / c^{2}, g_{\pi \pi}=0.23$, $g_{K K}=0.73[10]$.

The non-resonant amplitude $A_{\mathrm{nr}}$ is parametrized as $A_{\mathrm{nr}}=a_{1}^{\mathrm{nr}} e^{i \delta_{1}^{\mathrm{nr}}}+a_{2}^{\mathrm{nr}} e^{i \delta_{2}^{\mathrm{nr}}} s_{3}$, where $s_{3}=M^{2}\left(\pi^{+} \pi^{-}\right)\left(s_{3}\right.$ is not an independent variable and can be expressed via $s_{1}$ and $s_{2}$ but we use it here for clarity), $a_{1}^{\mathrm{nr}}, a_{2}^{\mathrm{nr}}, \delta_{1}^{\mathrm{nr}}$ and $\delta_{2}^{\mathrm{nr}}$ are free parameters of the fit.

The logarithmic likelihood function $\mathcal{L}$ is then constructed as

$$
\mathcal{L}=-2 \sum \log \left(f_{\text {sig }} S\left(s_{1}, s_{2}\right)+\left(1-f_{\text {sig }}\right) B\left(s_{1}, s_{2}\right)\right),
$$

where $S\left(s_{1}, s_{2}\right)$ is the density of signal events $\left|M\left(s_{1}, s_{2}\right)\right|^{2}$ convolved with the detector resolution function, $B\left(s_{1}, s_{2}\right)$ describes the combinatorial background that is considered to be constant and $f_{\text {sig }}$ is the fraction of signal events in the data sample. The fractions of signal events in the signal region are determined from fits to the $M_{\text {miss }}\left(\pi^{+} \pi^{-}\right)$spectrum and are found to be $0.937 \pm 0.015$ (stat.), $0.940 \pm$ 0.007 (stat.), $0.918 \pm 0.010$ (stat.) for final states with $\Upsilon(1 S), \Upsilon(2 S), \Upsilon(3 S)$, respectively. Both $S\left(s_{1}, s_{2}\right)$ and $B\left(s_{1}, s_{2}\right)$ are efficiency corrected.

In the fit to the data, we test the following assumptions on the spin and parity of the observed $Z_{b}$ states: $J^{P}=1^{+}$, $1^{-}, 2^{+}$and $2^{-}$. Note that $J^{P}=0^{+}$and $0^{-}$combinations are forbidden because of the observed $Z_{b} \rightarrow \Upsilon(n S) \pi$ and $Z_{b} \rightarrow h_{b}(m P) \pi$ decay modes, respectively. We find that the model with $J^{P}=1^{+}$assigned to both $Z_{b}$ states provides the best description of the data for all final states. Results of the fits to $\Upsilon(5 S) \rightarrow \Upsilon(n S) \pi^{+} \pi^{-}$signal events are shown in Fig. 3, where one-dimensional projections of the data and fits are compared. More details can be found in Ref. [9].

Analysis of quark composition of the initial and final states allows to state that $Z_{b}$ states should be comprised of (at least) four quarks. The proximity of the $Z_{b}(10610)$ and $Z_{b}(10650)$ masses to the sum of the $B$ and $B^{*}$ mesons and the sum of the two $B^{*}$ mesons, respectively, suggests that the decay rates of $Z_{b}$ states to these final states might be large. To search for $Z_{b} \rightarrow B^{(*)} \bar{B}^{*}$ decays we study threebody $e^{+} e^{-} \rightarrow B^{(*)} \bar{B}^{*} \pi$ transitions.

$B$ mesons are reconstructed in the following channels: $B^{+} \rightarrow J / \psi K^{+}, B^{+} \rightarrow \bar{D}^{0} \pi^{+}, B^{0} \rightarrow J / \psi K^{* 0}, B^{0} \rightarrow D^{-} \pi^{+}$, 

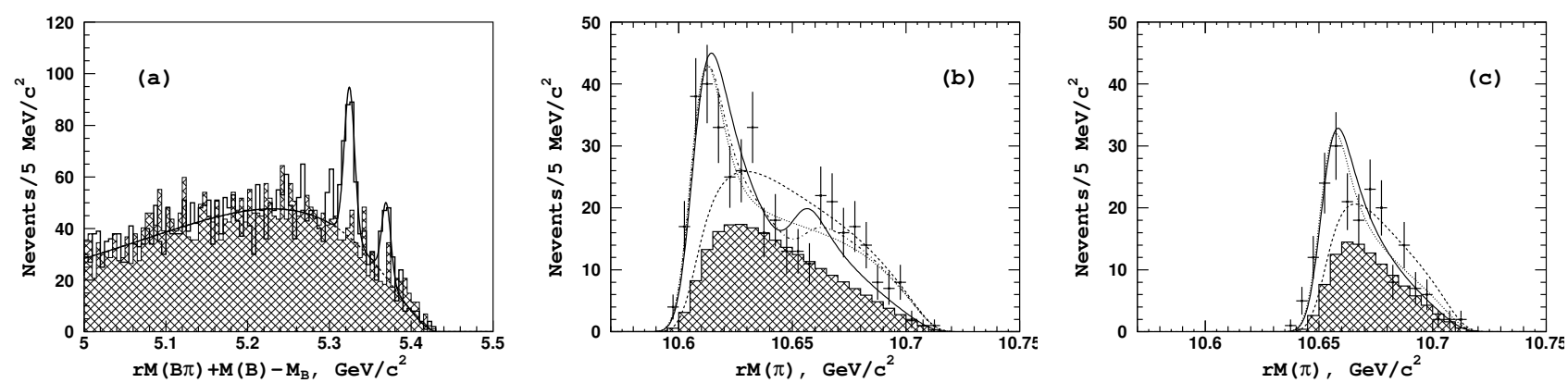

Figure 4. The (a) recoil mass distribution to $B \pi$ system and (b) pion recoil mass distribution for signal $B B^{*} \pi$ candidates, and (c) pionrecoil mass distribution for signal $B^{*} B^{*} \pi$ candidates. Points with error bars are data, solid line is the result of the fit with the nominal model (see text), dashed line - fit to pure non-resonant amplitude; dotted line - fit to one $Z_{b}$ state plus a non-resonant, and dash-dotted - two $Z_{b}$ states and non-resonant. Hatched histograms represent level of combinatorial background.

$B^{0} \rightarrow D^{*-} \pi^{+}$. We identify $B$ candidates by their invariant mass, $M(B)$, and momentum $P(B)$. We require $M(B)$ to be within 30 to $40 \mathrm{MeV} / c^{2}$ (depending on the $B$ decay mode) of the nominal $B$ mass. Neutral (charged) $D$ mesons originating from $B$ decays are reconstructed in the $\bar{D}^{0} \rightarrow K^{+} \pi^{-}$ and $\bar{D}^{0} \rightarrow K^{+} \pi^{+} \pi^{-} \pi^{-}\left(D^{-} \rightarrow K^{+} \pi^{-} \pi^{-}\right)$modes. Those originating from $D^{*-}$ decays are also reconstructed in the $\bar{D}^{0} \rightarrow K^{+} \pi^{-} \pi^{0}$ mode. To identify $D^{*-}$ candidates we require $\left|M\left(\overline{D^{0}} \pi^{-}\right)-M\left(\bar{D}^{0}\right)-0.14542\right|<2 \mathrm{MeV} / c^{2}$. The invariant mass of the $J / \psi \rightarrow \mu^{+} \mu^{-}$candidates is required to satisfy $\left|M\left(\mu^{+} \mu^{-}\right)-M_{J / \psi}\right|<30 \mathrm{MeV} / c^{2}$, where $M_{J / \psi}$ is the nominal mass of the $J / \psi$ meson. The $K^{* 0}$ is reconstructed in the $K^{* 0} \rightarrow K^{+} \pi^{-}$mode, the invariant mass of the $K^{* 0}$ candidate is required to be within $70 \mathrm{MeV} / c^{2}$ of the nominal $K^{* 0}$ mass. The invariant mass of a two-photon combination is required to be within $12 \mathrm{MeV} / c^{2}$ of the nominal $\pi^{0}$ mass.

Reconstructed $B^{+}$or $B^{0}$ candidates are then combined with a $\pi^{-}$candidate and a recoil mass to the $B \pi$ combination, $r M(B \pi)$, is calculated as $r M(B \pi)=\sqrt{E_{\mathrm{cms}}^{2}-P_{B \pi}^{2}}$, where $E_{\mathrm{cms}}$ is the center-of-mass energy, $P_{B \pi}$ - threemomentum of the $B \pi$ combination. Signal $\Upsilon(5 S) \rightarrow B B^{*} \pi$ events produce a narrow peak in the $r M(B \pi)$ spectrum around nominal $B^{*}$ mass, while $\Upsilon(5 S) \rightarrow B^{*} B^{*} \pi$ events produce a peak shifted to higher mass by about $45 \mathrm{MeV} / c^{2}$ due to a missed photon from the $B^{*} \rightarrow B \gamma$ decay. Figure 4(a) shows the $r M(B \pi)+M(B)-5.279 \mathrm{GeV} / c^{2}$ distribution for the experimental data. Use of $r M(B \pi)+M(B)-$ $5.279 \mathrm{GeV} / c^{2}$ instead of just $r M(B \pi)$ allows to remove correlations between $r M(B \pi)$ and $M(B)$ and improve resolution. It is important to note here that according to signal MC, $B B^{*} \pi$ events where the reconstructed $B$ is the one from $B^{*}$, produce a peak in the $r M(B \pi)$ distribution at the same position as signal events where the reconstructed $B$ is the prompt one.

For the subsequent analysis, we require $\mid(r M(B)+$ $M(B)-5.279)-M_{B^{*}} \mid<0.015 \mathrm{GeV} / c^{2}$ to select $\Upsilon(5 S) \rightarrow$ $B B^{*} \pi$ events and $\left|(r M(B)+M(B)-5.279)-\left(M_{B^{*}}+0.045\right)\right|<$ $0.015 \mathrm{GeV} / c^{2}$ to select $\Upsilon(5 S) \rightarrow B^{*} B^{*} \pi$ events. For selected candidate events we calculate mass recoiling against the charged pion: $r M(\pi)=\sqrt{E_{\mathrm{cms}}^{2}-P_{\pi}^{2}}$, where $P_{B \pi}$ - three-momentum of the charged pion. The $r M(\pi)$ distribution for candidates in the $B B^{*} \pi$ and $B^{*} B^{*} \pi$ signal regions are shown in Fig. 4(b) and (c), respectively. Excesses of signal events over the expected background levels are clearly visible at lower mass edges of the $r M(\pi)$ spectra. Distribution of signal events for the $\Upsilon(5 S) \rightarrow B B^{*} \pi$ decay is parametrized with the following model:

$$
S_{B B^{*} \pi}(m)=A_{Z_{b}(10610)}+A_{N R},
$$

where $A_{N R}$ is the non-resonant amplitude parametrized as just a complex constant and the $Z_{b}(10610)$ amplitudes is a Breit-Wigner function. We also apply a correction for reconstruction efficiency and phase space. As a variation of this nominal model, we also add a second Breit-Wigner amplitude to account for possible $Z_{b}(10650) \rightarrow B B^{*} \pi$ decay. We also fit the data with the $Z_{b}(10610)$ channel only. Results of these fits are shown in Fig. 4(b). Two models give about equally good description of the data: the nominal model and a model with an additional non-resonant amplitude. However, we select the former one as our nominal model since adding a non-resonant amplitude does a not improve the fit quality that much. The worst fit to the data is provided by a model with just non-resonant amplitude. From this analysis we estimate the significance of the $Z_{b}(10610) \rightarrow B B^{*}$ signal to exceed an $8 \sigma$ level.

As the nominal model for the $\Upsilon(5 S) \rightarrow B^{*} B^{*} \pi$ decay, we use the following parametrization:

$$
S_{B^{*} B^{*} \pi}(m)=A_{Z_{b}(10650)}+A_{N R}
$$

We also fit the data without a non-resonant component and with a non-resonant amplitude alone. Results of the fits are shown in Fig. 4(c).

The best description of the $B^{*} B^{*} \pi$ data is achieved by a model with the $Z_{b}(10650)$ amplitude only. Addition of a non-resonant amplitude does not provide any significant improvement of the fit quality. A fit with a nonresonant amplitude alone gives a much worse likelihood value. From this analysis, we estimate the significance of the $Z_{b}(10650) \rightarrow B^{*} B^{*}$ signal to be $6.8 \sigma$. 

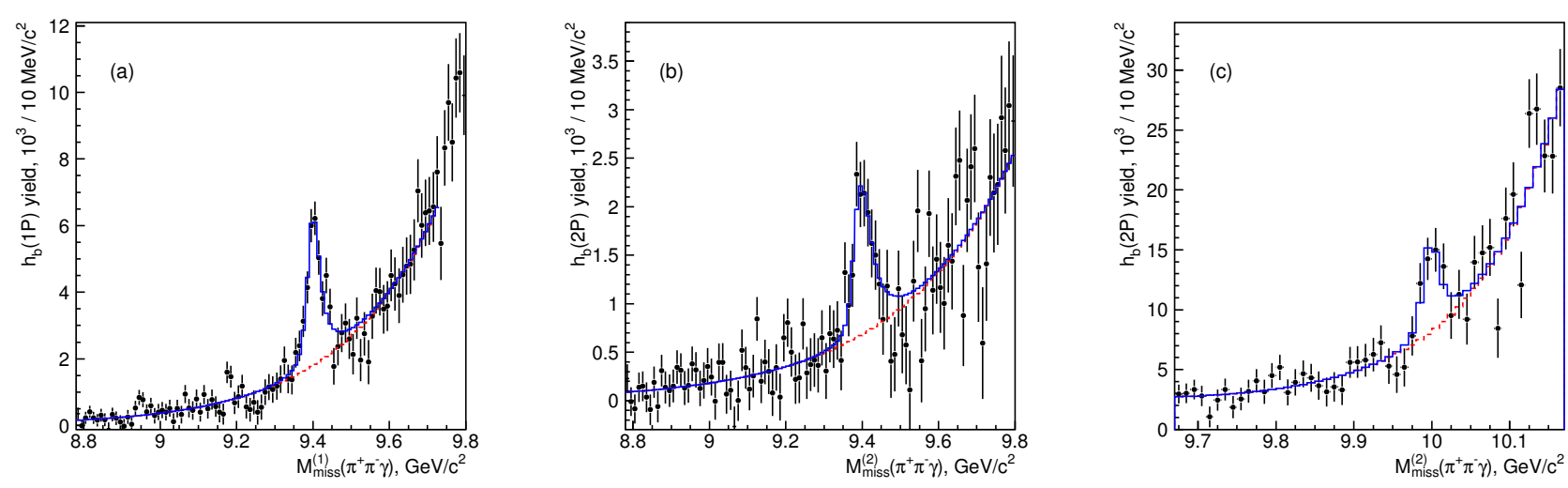

Figure 5. The $h_{b}(1 P)$ yield $v$ s. $M_{\text {miss }}^{(1)}\left(\pi^{+} \pi^{-} \gamma\right)\left(\right.$ a), and $h_{b}(2 P)$ yield $v s . M_{\text {miss }}^{(2)}\left(\pi^{+} \pi^{-} \gamma\right)$ in the $\eta_{b}(1 S)$ region (b) and in the $\eta_{b}(2 S)$ region (c). The solid (dashed) histogram presents the fit result (background component of the fit function).

\section{Evidence for the $\eta_{b}(2 S)$ and observation of $h_{b}(1,2 P) \rightarrow \eta_{b}(1 S) \gamma$}

To study the processes $e^{+} e^{-} \rightarrow \Upsilon(5 S) \rightarrow h_{b}(m P) \pi^{+} \pi^{-}$, $h_{b}(m P) \rightarrow \eta_{b}(n S) \gamma$ we reconstruct a $\pi^{+} \pi^{-}$pair and photon. The selection criteria are described in detail in Ref. [6]. The two-dimensional distribution $M_{\text {miss }}^{(m)}\left(\pi^{+} \pi^{-} \gamma\right) \equiv M_{\text {miss }}\left(\pi^{+} \pi^{-} \gamma\right)-M_{\text {miss }}\left(\pi^{+} \pi^{-}\right)+m_{h_{b}(m P)} v s$. $M_{\text {miss }}\left(\pi^{+} \pi^{-}\right)$contains a signal cluster at the location of two crossing bands. A band at $M_{\text {miss }}\left(\pi^{+} \pi^{-}\right)=m_{h_{b}(m P)}$ is due to events where the $\pi^{+} \pi^{-}$pair from the $e^{+} e^{-} \rightarrow h_{b}(m P) \pi^{+} \pi^{-}$ process is combined with a low energy combinatorial photon; a band at $M_{\text {miss }}^{(m)}\left(\pi^{+} \pi^{-} \gamma\right)=m_{\eta_{b}(n S)}$ is due to $e^{+} e^{-} \rightarrow$ $\eta_{b}(n S) \pi^{+} \pi^{-} \gamma$ events, not necessarily produced via $h_{b}(m P)$. We fit the $M_{\text {miss }}\left(\pi^{+} \pi^{-}\right)$spectra in $M_{\text {miss }}^{(m)}\left(\pi^{+} \pi^{-} \gamma\right)$ bins to measure the $h_{b}(m P)$ yield.

The results for the $h_{b}(1 P)$ and $h_{b}(2 P)$ yields as a function of $M_{\text {miss }}^{(m)}\left(\pi^{+} \pi^{-} \gamma\right)$ are presented in Fig. 5. Clear peaks at $9.4 \mathrm{GeV} / c^{2}$ and $10.0 \mathrm{GeV} / c^{2}$ are identified as signals for the $\eta_{b}(n S)$ and $\eta_{b}(2 S)$, respectively. Generic MC simulations indicate that no peaking backgrounds are expected in these spectra.

We fit the $h_{b}(m P)$ yield dependence on $M_{\text {miss }}^{(m)}\left(\pi^{+} \pi^{-} \gamma\right)$ to a sum of the $\eta_{b}(n S)$ signal components described by the convolution of a non-relativistic Breit-Wigner function with the resolution function and a background parametrized by an exponentiation of a first- [second-] order polynomial in the $\eta_{b}(1 S)\left[\eta_{b}(2 S)\right]$ region. The two $M_{\text {miss }}^{(m)}\left(\pi^{+} \pi^{-} \gamma\right)$ spectra [from the $h_{b}(1 P)$ and $h_{b}(2 P)$ ] with $\eta_{b}(1 S)$ signals are fitted simultaneously. We find event yields for the $h_{b}(m P) \rightarrow \eta_{b}(n S)$ transitions of $N_{1 P \rightarrow 1 S}=$ $(23.5 \pm 2.0) \times 10^{3}, N_{2 P \rightarrow 1 S}=(10.3 \pm 1.3) \times 10^{3}$ and $N_{2 P \rightarrow 2 S}=$ $(25.8 \pm 4.9) \times 10^{3}$; the fitted masses and width are $m_{\eta_{b}(1 S)}=$ $(9402.4 \pm 1.5 \pm 1.8) \mathrm{MeV} / c^{2}, \Gamma_{\eta_{b}(1 S)}=\left(10.8_{-3.7-2.0}^{+4.0}\right) \mathrm{MeV}$ and $m_{\eta_{b}(2 S)}=\left(9999.0 \pm 3.5_{-1.9}^{+2.8}\right) \mathrm{MeV} / c^{2}$. The confidence level of the $\eta_{b}(1 S)\left[\eta_{b}(2 S)\right]$ fit is $61 \%$ [36\%]. If the $\eta_{b}(2 S)$ width is allowed to float in the fit, we find $\Gamma_{\eta_{b}(2 S)}=\left(4_{-20}^{+12}\right) \mathrm{MeV}$ or $\Gamma_{\eta_{b}(2 S)}<24 \mathrm{MeV}$ at $90 \%$ C.L. using the Feldman-Cousins approach [11]. For mass and yield measurements, we fix the $\eta_{b}(2 S)$ width at its value from perturbative calculations [12] $\Gamma_{\eta_{b}(2 S)}=\Gamma_{\eta_{b}(1 S)} \frac{\Gamma_{e e}^{\mathrm{Y}(2 S)}}{\Gamma_{e e}^{\mathrm{Y}(1 S)}}=$ $\left(4.9_{-1.9}^{+2.7}\right) \mathrm{MeV}$, where the uncertainty is due to the experimental uncertainty in $\Gamma_{\eta_{b}(1 S)}$.

To estimate the systematic uncertainties in the $\eta_{b}(n S)$ parameters, we vary the polynomial orders and fit intervals in the $M_{\text {miss }}\left(\pi^{+} \pi^{-}\right) \& M_{\text {miss }}^{(m)}\left(\pi^{+} \pi^{-} \gamma\right)$ fits, and the $M_{\text {miss }}^{(m)}\left(\pi^{+} \pi^{-} \gamma\right)$ binning by scanning the starting point of the $10 \mathrm{MeV} / c^{2}$ bin with $1 \mathrm{MeV} / c^{2}$ steps. We also multiply the non-relativistic Breit-Wigner function by an $E_{\gamma}^{3}$ term expected for an electric dipole transition and include the uncertainty in the $h_{b}(1 P)$ and $h_{b}(2 P)$ masses and in the estimated value of the $\eta_{b}(2 S)$ width. We add the various contributions in quadrature to estimate the total systematic uncertainty. For the hyperfine splittings $m_{\Upsilon(n S)}-m_{\eta_{b}(n S)}$ we determine $\Delta M_{\mathrm{HF}}(1 S)=(57.9 \pm 2.3) \mathrm{MeV} / c^{2}$ and $\Delta M_{\mathrm{HF}}(2 S)=\left(24.3_{-4.5}^{+4.0}\right) \mathrm{MeV} / c^{2}$, where statistical and systematic uncertainties in mass are added in quadrature.

Using Wilks' theorem, we find $15 \sigma[9 \sigma]$ for the $h_{b}(1 P) \rightarrow \eta_{b}(1 S) \gamma\left[h_{b}(2 P) \rightarrow \eta_{b}(1 S) \gamma\right]$ statistical significance. For the significance of the $\eta_{b}(2 S)$ signal, we use a method [13] that requires definition of the search window to take into account the "look elsewhere effect." For the ratio $r=\frac{\Delta M_{\mathrm{HF}}(2 S)}{\Delta M_{\mathrm{HF}}(1 S)}$, perturbative calculations [14] pre$\operatorname{dict} \frac{m_{\Upsilon(2 S)}^{2}}{m_{\Upsilon(1 S)}^{2}} \frac{\Gamma_{e e}^{r(2 S)}}{\Gamma_{e e}^{r(1 S)}}=0.513 \pm 0.011$ (where the error is due to the uncertainties in $\Gamma_{e e}$ ); this is consistent with the measured value of $0.420_{-0.079}^{+0.071}$. To determine boundaries of the search window, we conservatively assume $r=0$ and $r=1$. We find the significance of the $\eta_{b}(2 S)$ signal to be $4.8 \sigma$ (4.2 $\sigma$ including systematics).

We measure $\mathcal{B}\left[h_{b}(1 P) \rightarrow \eta_{b}(1 S) \gamma\right]=(49.2 \pm$ $\left.5.7_{-3.3}^{+5.6}\right) \%, \mathcal{B}\left[h_{b}(2 P) \rightarrow \eta_{b}(1 S) \gamma\right]=\left(22.3 \pm 3.8_{-3.3}^{+3.1}\right) \%$ and $\mathcal{B}\left[h_{b}(2 P) \rightarrow \eta_{b}(2 S) \gamma\right]=\left(47.5 \pm 10.5_{-7.7}^{+6.8}\right) \%$.

\section{Conclusion}

In summary, we have observed the $P$-wave spin-singlet bottomonium states $h_{b}(1 P)$ and $h_{b}(2 P)$ in the reaction $e^{+} e^{-} \rightarrow \Upsilon(5 S) \rightarrow h_{b}(m P) \pi^{+} \pi^{-}$. The measured hyperfine splittings are consistent with zero as expected. A detailed analysis revealed that $h_{b}(m P)$ states in $\Upsilon(5 S)$ decays are dominantly produced via intermediate charged bottomonium-like resonances $Z_{b}(10610)$ and $Z_{b}(10650)$. 
Both these resonances have also been observed in $\Upsilon(n S) \pi$ spectra in the $\Upsilon(5 S) \rightarrow \Upsilon(n S) \pi^{+} \pi^{-}$decays. Weighted averages over all five channels give $M=10607.2 \pm$ $2.0 \mathrm{MeV} / c^{2}, \Gamma=18.4 \pm 2.4 \mathrm{MeV}$ for the $Z_{b}(10610)$ and $M=10652.2 \pm 1.5 \mathrm{MeV} / c^{2}, \Gamma=11.5 \pm 2.2 \mathrm{MeV}$ for the $Z_{b}(10650)$, where statistical and systematic errors are added in quadrature. The relative phase between $Z_{b}(10610)$ and $Z_{b}(10650)$ is consistent with zero for the final states with the $\Upsilon(n S)$ and consistent with 180 degrees for the final states with $h_{b}(m P)$. Amplitude analysis strongly favors the $J^{P}=1^{+}$spin-parity assignment for both the $Z_{b}(10610)$ and $Z_{b}(10650)$ states. Since the $\Upsilon(5 S)$ has negative $G$-parity, the $Z_{b}$ states have positive $G$-parity due to the emission of the pion. We also observe significant signals of $Z_{b}(10610)$ and $Z_{b}(10650)$ states in $B B^{*}$ and $B^{*} B^{*}$ final states, respectively.

We report the first evidence for the $\eta_{b}(2 S)$ using the $h_{b}(2 P) \rightarrow \eta_{b}(2 S) \gamma$ transition, with a significance, including systematics, of $4.2 \sigma$, and the first observation of the $h_{b}(1 P) \rightarrow \eta_{b}(1 S) \gamma$ and $h_{b}(2 P) \rightarrow \eta_{b}(1 S) \gamma$ transitions. The mass and width parameters of the $\eta_{b}(1 S)$ and $\eta_{b}(2 S)$ are measured to be $m_{\eta_{b}(1 S)}=(9402.4 \pm 1.5 \pm 1.8) \mathrm{MeV} / c^{2}$, $m_{\eta_{b}(2 S)}=\left(9999.0 \pm 3.5_{-1.9}^{+2.8}\right) \mathrm{MeV} / c^{2}$ and $\Gamma_{\eta_{b}(1 S)}=$ $\left(10.8_{-3.7-2.0}^{+4.0}+4.5 \mathrm{MeV}\right.$. The $m_{\eta_{b}(2 S)}$ and $\Gamma_{\eta_{b}(1 S)}$ are first measurements; the $m_{\eta_{b}(1 S)}$ measurement is the most precise and is about $10 \mathrm{MeV} / c^{2}$ higher than the current world average [7]. The hyperfine splittings, $\Delta M_{\mathrm{HF}}(1 S)=(57.9 \pm$ 2.3) $\mathrm{MeV} / c^{2}$ and $\Delta M_{\mathrm{HF}}(2 S)=\left(24.3_{-4.5}^{+4.0}\right) \mathrm{MeV} / c^{2}$, are in agreement with theoretical calculations [14]. We measure branching fractions for the transitions $\mathcal{B}\left[h_{b}(1 P) \rightarrow\right.$ $\left.\eta_{b}(1 S) \gamma\right]=\left(49.2 \pm 5.7_{-3.3}^{+5.6}\right) \%, \mathcal{B}\left[h_{b}(2 P) \rightarrow \eta_{b}(1 S) \gamma\right]=$ $\left(22.3 \pm 3.8_{-3.3}^{+3.1}\right) \%$ and $\mathcal{B}\left[h_{b}(2 P) \rightarrow \eta_{b}(2 S) \gamma\right]=(47.5 \pm$ $\left.10.5_{-7.7}^{+6.8}\right) \%$ that are somewhat higher than theoretical expectations [1]. We update the $h_{b}(1 P)$ and $h_{b}(2 P)$ mass measurements $m_{h_{b}(1 P)}=(9899.1 \pm 0.4 \pm 1.0) \mathrm{MeV} / c^{2}$, $m_{h_{b}(2 P)}=(10259.8 \pm 0.5 \pm 1.1) \mathrm{MeV} / c^{2}$, and $1 P$ and $2 P$ hyperfine splittings $\Delta M_{\mathrm{HF}}(1 P)=(+0.8 \pm 1.1) \mathrm{MeV} / c^{2}$, $\Delta M_{\mathrm{HF}}(2 P)=(+0.5 \pm 1.2) \mathrm{MeV} / c^{2}$.

We thank the KEKB group for excellent operation of the accelerator; the KEK cryogenics group for efficient solenoid operations; and the KEK computer group, the NII, and PNNL/EMSL for valuable computing and SINET4 network support. We acknowledge support from MEXT, JSPS and Nagoya's TLPRC (Japan); ARC and DI-
ISR (Australia); NSFC (China); MSMT (Czechia); DST (India); INFN (Italy); MEST, NRF, GSDC of KISTI, and WCU (Korea); MNiSW (Poland); MES and RFAAE (Russia); ARRS (Slovenia); SNSF (Switzerland); NSC and MOE (Taiwan); and DOE and NSF (USA).

\section{References}

[1] S. Godfrey and J. L. Rosner, Phys. Rev. D 66, 014012 (2002).

[2] T. K. Pedlar et al. (CLEO Collaboration), Phys. Rev. Lett. 107, 041803 (2011).

[3] K.-F. Chen et al. (Belle Collaboration), Phys. Rev. Lett. 100, 112001 (2008).

[4] A. Abashian et al. (Belle Collaboration), Nucl. Instrum. Methods Phys. Res., Sect. A 479, 117 (2002).

[5] S. Kurokawa and E. Kikutani, Nucl. Instrum. Methods Phys. Res. Sect. A 499, 1 (2003), and other papers included in this Volume.

[6] R. Mizuk, D. M. Asner, A. Bondar, T. K. Pedlar, et al. (Belle Collaboration), Phys. Rev. Lett. 109, 232002 (2012).

[7] J. Beringer et al. et al. (Particle Data Group), Phys. Rev. D 86, 010001 (2012).

[8] A. Bondar, A. Garmash, R. Mizuk, D. Santel, K. Kinoshita et al. (Belle Collaboration), Phys. Rev. Lett. 108, 122001 (2012).

[9] I. Adachi et al. (Belle Collaboration), arXiv:1209.6450.

[10] A. Garmash et al. (Belle Collaboration), Phys. Rev. Lett. 96, 251803 (2006).

[11] G. J. Feldman and R. D. Cousins, Phys. Rev. D 57, 3873 (1998).

[12] W. Kwong et al., Phys. Rev. D 37, 3210 (1988) (and references therein); R. Barbieri et al., Nucl. Phys. B 154, 535 (1979).

[13] E. Gross and O. Vittels, Eur. Phys. J. C 70, 525 (2010).

[14] R. Van Royen and V. F. Weisskopf, Nuovo Cim. A 50, 617 (1967) [Erratum-ibid. A 51, 583 (1967)]; A. De Rujula, H. Georgi and S. L. Glashow, Phys. Rev. D 12, 147 (1975). 